\title{
THE EIGENVALUE PROBLEM FOR A CLASS OF LONG, THIN ELASTIC STRUCTURES WITH PERIODIC GEOMETRY
}

\author{
BY \\ ROBERT E. MILLER \\ Center for Applied Mathematical Sciences, University of Southern California, Los Angeles, California
}

\begin{abstract}
Elastic structures consisting of many thin elements arranged periodically (such as grids and trusses) are common in applications. Using standard numerical techniques such as splines in attempting to analyze these structures leads to serious difficulties due to the complicated geometry. Instead, one can use methods of asymptotic analysis to derive a "simple" problem whose solution approximates that of the original problem. In this paper we begin with a linearized elastic system on a threedimensional domain with two of the dimensions small relative to the third and derive a one-dimensional eigenvalue problem by letting a small parameter tend to zero. The resulting equation has coefficients which vary periodically with the spatial variable, so we let the period tend to zero to obtain the "homogenized" equation which has constant coefficients whose values can be easily calculated once the geometry of the structure is specified. We illustrate with several examples.
\end{abstract}

1. Introduction. In this paper we consider the eigenvalue problem for a class of structures with one dimension large compared to the others. In particular, we consider structures contained in the cylinder $Q^{e}=\omega^{e} \times(0, L)$ where $\omega^{e}$ is a region in $\mathbf{R}^{2}$ whose diameter depends on the (small) parameter $e$. The results obtained in this paper are applicable to structures with a wide range of cross sections. In the case of a rectangular cross section, for example, we will take $\omega^{e}=\left(-a_{1} e, a_{1} e\right) \times\left(-a_{2} e, a_{2} e\right)$. In any case the centroid of $\omega^{e}$ must lie at the origin. Let $\Omega_{\delta \varepsilon}^{e}$ denote the part of $Q^{e}$ occupied by material, and suppose that $\Omega_{\delta \varepsilon}^{e}$ has period $c \varepsilon$; i.e., $\Omega_{\delta \varepsilon}^{e}$ is composed of $N$ identical sections where $N=L / c \varepsilon$. The parameter $\delta$, roughly speaking, indicates the amount of material in the structure, and the parameters $a_{1}, a_{2}$, and $c$ are included to simplify the calculation of the coefficients in the final result (see Sec. 3.2). As an example, consider the structure depicted in Fig. 1a which consists of four vertical bars of length $L$ and square cross section $e \delta / 2 \times e \delta / 2$. The vertical

Received January 2, 1992.

1991 Mathematics Subject Classification. Primary 35B27.

This research was supported in part by the Air Force Office of Scientific Research under grant AFOSR-900091 and in part by the National Aeronautics and Space Administration under NASA grant NAG-1-1116. Current address: Department of Mathematical Sciences, University of Arkansas, Fayetteville, Arkansas 72701.

(C)1994 Brown University 
bars are separated by $N+1$ periodically distributed horizontal bars of rectangular cross section $e \delta / 2 \times \varepsilon \delta$ (except for the bars on the top and bottom bases for which the cross section is $e \delta / 2 \times \varepsilon \delta / 2)$ where $\varepsilon=L / N$. A representative period for this structure is shown in Fig. $1 \mathrm{~b}$.

Since the parameter $e$ is assumed to be small relative to the length $L$, we expect that the behavior of our structure somehow resembles the behavior of a beam. To show that this is indeed the case, we will show that the solutions of the threedimensional eigenvalue problem converge to solutions of a one-dimensional problem as $e \rightarrow 0$. Since the domain $Q^{e}$ depends on $e$, we transform the problem to a fixed domain by an appropriate scaling of the variables (see Sec. 2) before taking the limit. We will use $\mathbf{x}^{e}=\left(x_{1}^{e}, x_{2}^{e}, x_{3}^{e}\right)$ to denote a point in $Q^{e}$ and $\mathbf{x}=\left(x_{1}, x_{2}, x_{3}\right)$ to denote a point in the fixed domain $Q=Q^{1}$. When we take the limit in $e$ we will obtain a one-dimensional problem in which the coefficients are periodic functions of $\varepsilon$. Hence, we will let $\varepsilon$ tend to zero to obtain the "homogenized" equation. Finally, we will take a third limit (by letting $\delta \rightarrow 0$ ) to facilitate the computation of the explicit values of the coefficients (which will depend on the geometry). The results of this paper rely heavily upon the work of D. Cioranescu and J. Saint Jean Paulin (see $[8,9])$ who considered the static case. Cimetière et al. used similar techniques to study nonlinear rods in [5].

Let $T_{\delta \varepsilon}^{e}$ be the part of $Q^{e}$ not occupied by material (i.e., $T_{\delta \varepsilon}^{e}=Q^{e} \backslash \Omega_{\delta \varepsilon}^{e}$ ),

$$
Y_{\delta \varepsilon}^{e}=\left\{\left(x_{1}^{e}, x_{2}^{e}, x_{3}^{e}\right) \in \Omega_{\delta \varepsilon}^{e} \mid 0<x_{3}^{e}<c \varepsilon\right\},
$$

and

$$
S_{\delta \varepsilon}^{e}\left(x_{3}^{e}\right)=\left\{\left(x_{1}^{e}, x_{2}^{e}\right) \mid\left(x_{1}^{e}, x_{2}^{e}, x_{3}^{e}\right) \in Y_{\delta \varepsilon}^{e}\right\}
$$

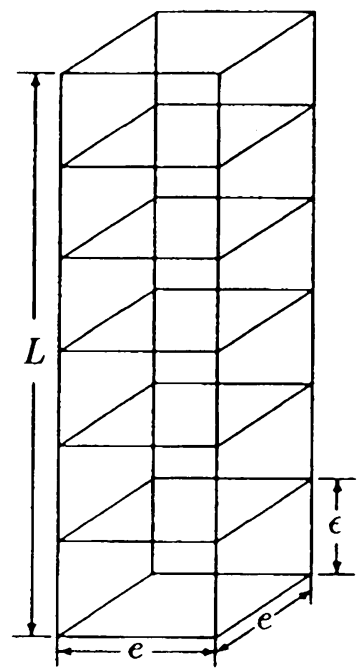

(a) The truss

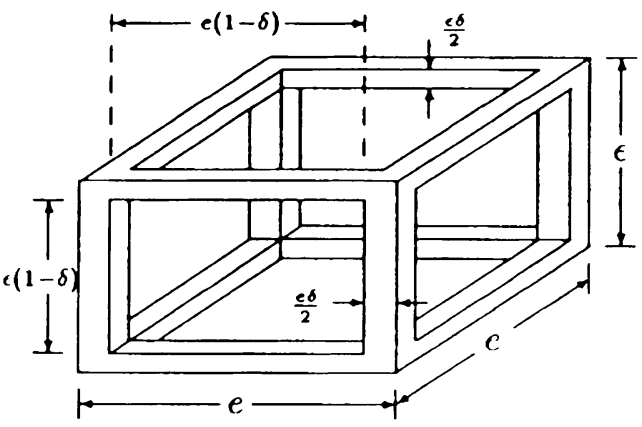

(b) The representative period

Fig. 1. A structure with $a_{1}=a_{2}=1 / 2$ and $c=1$ 


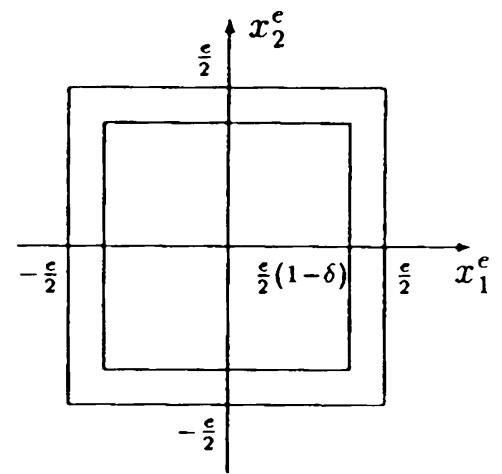

(a) $0<x_{3}^{e}<\frac{\varepsilon \delta}{2}$ or $\varepsilon\left(1-\frac{\delta}{2}\right)<x_{3}^{e}<\varepsilon$

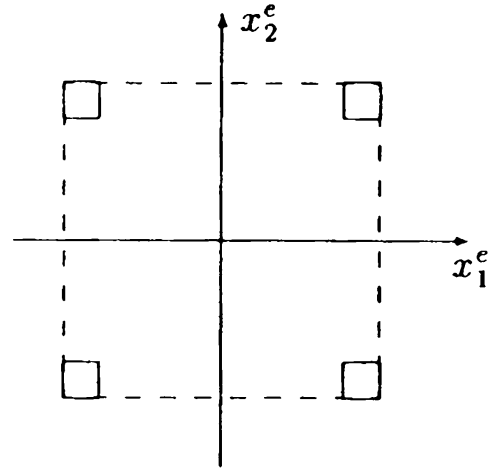

(b) $\frac{\varepsilon \delta}{2}<x_{3}^{e}<\varepsilon\left(1-\frac{\delta}{2}\right)$

FIG. 2. The set $S_{\delta \varepsilon}^{e}\left(x_{3}^{e}\right)$

The set $S_{\delta \varepsilon}^{e}\left(x_{3}^{e}\right)$ corresponding to the structure in Fig. 1 is shown in Fig. 2. We can extend $S_{\delta \varepsilon}^{e}$ by periodicity to all of $(0, L)$. Finally, define

$$
\Gamma_{\delta}^{e}=S_{\delta \varepsilon}^{e}(0)=S_{\delta \varepsilon}^{e}(c \varepsilon)
$$

which is independent of $\varepsilon$.

Generally we will consider structures in which the material is concentrated on the faces of the period or along the edges. The only restriction we place upon the geometry of the structure is that it satisfy

$$
\int_{S_{\delta \varepsilon}^{e}\left(x_{3}^{e}\right)} x_{1}^{e} d x_{1}^{e} d x_{2}^{e}=\int_{S_{\delta \varepsilon}^{e}\left(x_{3}^{e}\right)} x_{2}^{e} d x_{1}^{e} d x_{2}^{e}=\int_{S_{\delta \varepsilon}^{e}\left(x_{3}^{e}\right)} x_{1}^{e} x_{2}^{e} d x_{1}^{e} d x_{2}^{e}=0,
$$

for all $0<x_{3}^{e}<L$. This assumption clearly holds for the structure shown in Fig. 1. The representative periods of other structures for which (1) holds are shown in Figs. 3 and 4.

In what follows we make use of the summation convention on repeated indices. Latin indices (e.g., $i, j, k)$ take values in the set $\{1,2,3\}$ and Greek indices $(\alpha, \beta$, etc.) in $\{1,2\}$. For simplicity of notation we will suppress the $\varepsilon$ and $\delta$ for now, and we will drop the $e$ whenever $e=1$. We also adopt the following notation for
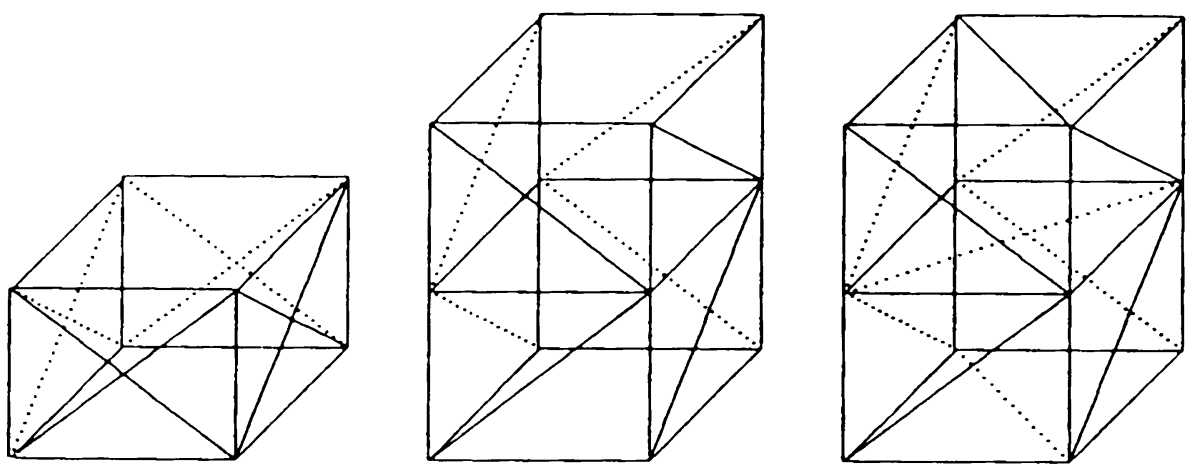

FIG. 3. Representative periods of other trusses 


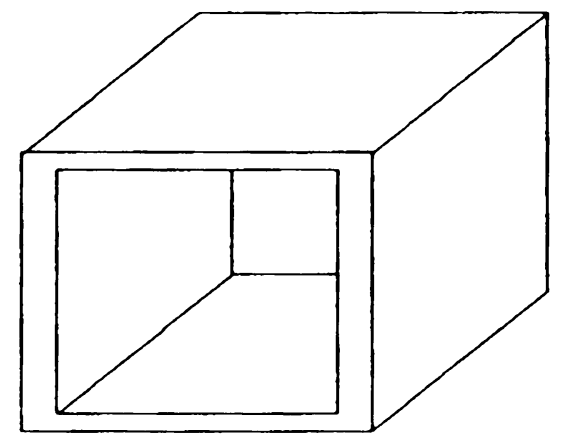

Fig. 4. An example of a period for a tower

norms: Let $\Omega$ be an open subset of $\mathbf{R}^{n}$. Then for $v \in H^{m}(\Omega)$,

and

$$
|v|_{m, \Omega}=\left(\int_{\Omega} \sum_{|\alpha|=m}\left|D^{\alpha} v\right|^{2} d x\right)^{1 / 2},
$$

$$
\|v\|_{m, \Omega}=\left(\int_{\Omega} \sum_{|\alpha| \leq m}\left|D^{\alpha} v\right|^{2} d x\right)^{1 / 2},
$$

where $D^{\alpha} v=\partial^{|\alpha|} v / \partial x_{1}^{\alpha_{1}} \ldots \partial x_{n}^{\alpha_{n}}$ for the multi-index $\alpha=\left(\alpha_{1}, \ldots, \alpha_{n}\right)$. We also use the same notation for norms on spaces of vector-valued functions. Observe that for $m=0,|\cdot|_{0, \Omega}=\|\cdot\|_{0, \Omega}$, and both symbols denote the usual $L^{2}$ norm. Finally, we denote the measure of a set $E$ by $|E|$. It will always be clear from the context what the symbol $|\cdot|$ means.

The stress tensor $\boldsymbol{\sigma}^{e}$ and the displacement $\mathbf{u}^{e}$ are related by

where

$$
\sigma_{i j}^{e}=a_{i j k h} \gamma_{k h}^{e}\left(\mathbf{u}^{e}\right)
$$

$$
\gamma_{k h}^{e}\left(\mathbf{v}^{e}\right)=\frac{1}{2}\left(\partial_{h}^{e} v_{k}^{e}+\partial_{k}^{e} v_{h}^{e}\right)
$$

is the linearized strain tensor and $\partial_{h}^{e} v^{e}=\partial v^{e} / \partial x_{h}^{e}$.

We make the following symmetry and coercivity assumptions on the elasticity coefficients $a_{i j k h}$ :

(i) $a_{i j k h}=a_{j i k h}=a_{k h i j}$ for all $i, j, k, h$.

(ii) There exists $\alpha_{0}>0$ such that $\alpha_{0} v_{i j} v_{i j} \leq a_{i j k h} v_{k h} v_{i j}$

for all $\mathbf{v}=\left\{v_{i j}\right\}$ with $v_{i j}=v_{j i}$.

Let $\mathbf{w}^{e}=\left(w_{i}^{e}\right): \bar{\Omega}^{e} \times(0, \infty) \rightarrow \mathbf{R}^{3}$ denote the displacement field. Set $\Gamma_{0}^{e}=$ $\Gamma^{e} \times\{0\}$ and $\Gamma_{1}^{e}=\partial \bar{\Omega}^{e} \backslash \Gamma_{0}^{e}$. Then for all $t>0, \mathbf{w}^{e}$ satisfies

$$
\begin{aligned}
& \rho^{e} \frac{\partial^{2} w_{i}^{e}}{\partial t^{2}}-\partial_{j}^{e}\left(a_{i j k h} \gamma_{k h}^{e}\left(\mathbf{w}^{e}\right)\right)=0 \text { in } \bar{\Omega}^{e}, \\
& \mathbf{w}^{e}=0 \text { on } \Gamma_{0}^{e}, \\
& a_{i j k h} \gamma_{k h}^{e}\left(\mathbf{w}^{e}\right) n_{j}=0 \text { on } \Gamma_{1}^{e} \text {, }
\end{aligned}
$$


where $\rho^{e}$ denotes the mass of the material, $\mathbf{n}=\left(n_{j}\right)$ denotes the outward unit normal, and we have assumed there are no applied body or surface forces. We seek a solution of the form

$$
\mathbf{w}^{e}\left(\mathbf{x}^{e}, t\right)=\mathbf{u}^{e}\left(\mathbf{x}^{e}\right) \cos \omega^{e} t \quad \text { or } \quad \mathbf{w}^{e}\left(\mathbf{x}^{e}, t\right)=\mathbf{u}^{e}\left(\mathbf{x}^{e}\right) \sin \omega^{e} t .
$$

Set $\Lambda^{e}=\left(\omega^{e}\right)^{2}$. Then the problem reduces to finding real numbers $\Lambda^{e}$ and functions $\mathbf{u}^{e}: \overline{\mathbf{\Omega}}^{e} \rightarrow \mathbf{R}^{3}$ that solve

Define the spaces $\mathbf{V}^{e}$ and $\mathbf{H}^{e}$ by

$$
\begin{aligned}
& -\partial_{j}^{e} \sigma_{i j}^{e}\left(\mathbf{u}^{e}\right)=\rho^{e} \Lambda^{e} u_{i}^{e} \quad \text { in } \Omega^{e}, \\
& \mathbf{u}^{e}=0 \text { on } \Gamma_{0}^{e} \text {, } \\
& \sigma_{i j}^{e}\left(\mathbf{u}^{e}\right) n_{j}=0 \text { on } \Gamma_{1}^{e} \text {. }
\end{aligned}
$$

$$
\begin{gathered}
\mathbf{v}^{e}=\left\{\mathbf{v}^{e}=\left(v_{i}^{e}\right) \in \mathbf{H}^{1}\left(\boldsymbol{\Omega}^{e}\right) \mid \mathbf{v}^{e}=0 \text { on } \Gamma_{0}^{e}\right\}, \\
\mathbf{H}^{e}=\mathbf{L}^{2}\left(\boldsymbol{\Omega}^{e}\right),
\end{gathered}
$$

and the bilinear forms $B^{e}(\cdot, \cdot)$ on $\mathbf{V}^{e}$ and $N^{e}(\cdot, \cdot)$ on $\mathbf{H}^{e}$ by

$$
\begin{gathered}
B^{e}\left(\mathbf{u}^{e}, \mathbf{v}^{e}\right)=\int_{\Omega^{e}} a_{i j k h} \gamma_{k h}^{e}\left(\mathbf{u}^{e}\right) \gamma_{i j}^{e}\left(\mathbf{v}^{e}\right) d x^{e}, \\
N^{e}\left(\mathbf{u}^{e}, \mathbf{v}^{e}\right)=\int_{\Omega^{e}} \rho^{e} u_{i}^{e} v_{i}^{e} d x^{e} .
\end{gathered}
$$

By the symmetry of the $a_{i j k h}$, the eigenvalue problem (7) can be written in variational form as follows. Find $\left(\Lambda^{e}, \mathbf{u}^{e}\right) \in \mathbf{R} \times \mathbf{V}^{e}$ such that

$$
B^{e}\left(\mathbf{u}^{e}, \mathbf{v}^{e}\right)=\Lambda^{e} N^{e}\left(\mathbf{u}^{e}, \mathbf{v}^{e}\right) \text { for all } \mathbf{v}^{e} \in \mathbf{V}^{e} .
$$

By the ellipticity of the $a_{i j k h}((4)(i i))$ and the compactness of the embedding $\mathbf{V}^{e} \hookrightarrow$ $\mathbf{H}^{e}$ (by the Rellich Theorem, see $[2,16]$ ), the eigenvalues $\Lambda^{e}$ are positive and can be ordered so that

$$
0<\Lambda^{e, 1} \leq \Lambda^{e, 2} \leq \cdots \leq \Lambda^{e, l} \leq \cdots,
$$

with $\lim _{l \rightarrow \infty} \Lambda^{e, l}=+\infty$. Each eigenvalue has finite multiplicity, and there exist eigenfunctions $\mathbf{u}^{e, l} \in \mathbf{V}^{e}$ such that

$$
B^{e}\left(\mathbf{u}^{e, l}, \mathbf{v}^{e}\right)=\Lambda^{e, l} N^{e}\left(\mathbf{u}^{e, l}, \mathbf{v}^{e}\right) \quad \text { for all } \mathbf{v}^{e} \in \mathbf{V}^{e},
$$

and the $\mathbf{u}^{e, l}$ can be normalized so that

$$
\int_{\Omega^{e}} \rho^{e} \mathbf{u}^{e, l} \cdot \mathbf{u}^{e, k} d x^{e}=e^{2} \delta_{l k}
$$

(See [3, Sec. 5.1; 12].) Consider the Rayleigh quotient

$$
J^{e}\left(\mathbf{v}^{e}\right)=B^{e}\left(\mathbf{v}^{e}, \mathbf{v}^{e}\right) / N^{e}\left(\mathbf{v}^{e}, \mathbf{v}^{e}\right) .
$$

Let $\mathscr{V}_{l}^{e}$ denote the family of all subspaces of $\mathbf{v}^{e}$ of dimension $l$. Then we have the following "min-max" characterization of the $l$ th eigenvalue $\Lambda^{e, l}$ (see [3, p. 170; 15]):

$$
\Lambda^{e, l}=\min _{U^{e} \in \mathscr{V}_{l}^{e}}\left\{\max _{\mathbf{v}^{e} \in U^{e}} J^{e}\left(\mathbf{v}^{e}\right)\right\} .
$$


2. Derivation of the beam eigenvalue problem. For simplicity we will assume that the structure has rectangular cross section with the period shown in Fig. 1b, but all results obtained in this section will also hold for the more general case discussed at the beginning of the paper. The techniques of this section were used by Ciarlet and Kesavan to derive the eigenvalue problem for a plate in [4]. (See also [3] and the references therein.) We first transform the eigenvalue problem given by (12) to the fixed domain $\Omega \subset Q=(-1 / 2,1 / 2) \times(-1 / 2,1 / 2) \times(0, L)$ as follows: With each point $\mathbf{x} \in \bar{Q}$, we associate the point $\boldsymbol{\pi}^{e} \mathbf{x}=\mathbf{x}^{e} \in \bar{Q}^{e}$ by

$$
\boldsymbol{\pi}^{e}: \mathbf{x}=\left(x_{1}, x_{2}, x_{3}\right) \mapsto \mathbf{x}^{e}=\left(e x_{1}, e x_{2}, x_{3}\right) .
$$

We define $\mathbf{u}(e)$ and $\Lambda(e)$ by the scalings

$$
\Lambda(e)=\Lambda^{e},
$$

and

$$
\left.\begin{array}{l}
u_{\alpha}^{e}\left(\mathbf{x}^{e}\right)=e u_{\alpha}(e)(\mathbf{x}) \\
u_{3}^{e}\left(\mathbf{x}^{e}\right)=e^{2} u_{3}(e)(\mathbf{x})
\end{array}\right\} \quad \text { for all } \mathbf{x}^{e}=\pi^{e} \mathbf{x} \in \bar{\Omega}^{e} .
$$

We also scale the density by

$$
\rho^{e}=e^{2} \rho
$$

where $\rho$ is a constant independent of $e$. Any function $\mathbf{v}^{e}$ defined on $\bar{\Omega}^{e}$ can be scaled in the same way as $\mathbf{u}^{e}$ given by (18). We remark that these scalings are mainly for mathematical convenience. The only physical significance lies in the fact that as $e$ tends to 0 , we expect the displacements $u_{\alpha}^{e}$ to be larger than the displacement $u_{3}^{e}$.

With $\partial_{i}=\partial / \partial x_{i}$ and $\gamma_{i j}(\mathbf{v})=\frac{1}{2}\left(\partial_{i} v_{j}+\partial_{j} v_{i}\right)$, it is easy to check that

$$
\begin{gathered}
d x^{e}=e^{2} d x, \\
\partial_{\beta}^{e} v_{\alpha}^{e}=\partial_{\beta} v_{\alpha}, \quad \partial_{3}^{e} v_{\alpha}^{e}=e \partial_{3} v_{\alpha}, \\
\partial_{\beta}^{e} v_{3}^{e}=e \partial_{\beta} v_{3}, \quad \partial_{3}^{e} v_{3}^{e}=e^{2} \partial_{3} v_{3},
\end{gathered}
$$

and

$$
\gamma_{\alpha \beta}^{e}\left(\mathbf{v}^{e}\right)=\gamma_{\alpha \beta}(\mathbf{v}), \quad \gamma_{\alpha 3}^{e}\left(\mathbf{v}^{e}\right)=e \gamma_{\alpha 3}(\mathbf{v}), \quad \gamma_{33}^{e}\left(\mathbf{v}^{e}\right)=e^{2} \gamma_{33}(\mathbf{v}) .
$$

Thus, the scaled unknowns $u(e)$ and $\Lambda(e)$ solve

$$
\begin{aligned}
& \Lambda(e)>0, \quad \mathbf{u}(e) \in \mathbf{V}=\left\{\mathbf{v} \in \mathbf{H}^{1}(\Omega) \mid \mathbf{v}=0 \text { on } \Gamma_{0}\right\} \\
& B(e)(\mathbf{u}(e), \mathbf{v})=\Lambda(e) N(e)(\mathbf{u}(e), \mathbf{v}) \text { for all } \mathbf{v} \in \mathbf{V},
\end{aligned}
$$

where

$$
\begin{aligned}
B(e)(\mathbf{u}, \mathbf{v})=\int_{\Omega} & \left\{e^{-4} a_{\alpha \beta \tau \eta} \gamma_{\tau \eta}(\mathbf{u}) \gamma_{\alpha \beta}(\mathbf{v})+2 e^{-3}\left[a_{\alpha \beta \tau 3} \gamma_{\tau 3}(\mathbf{u}) \gamma_{\alpha \beta}(\mathbf{v})+a_{\alpha 3 \tau \eta} \gamma_{\tau \eta}(\mathbf{u}) \gamma_{\alpha 3}(\mathbf{v})\right]\right. \\
& +e^{-2}\left[a_{\alpha \beta 33} \gamma_{33}(\mathbf{u}) \gamma_{\alpha \beta}(\mathbf{v})+4 a_{\alpha 3 \tau} \gamma_{\tau 3}(\mathbf{u}) \gamma_{\alpha 3}(\mathbf{v})+a_{33 \tau \eta} \gamma_{\tau \eta}(\mathbf{u}) \gamma_{33}(\mathbf{v})\right] \\
& \left.+2 e^{-1}\left[a_{\alpha 333} \gamma_{33}(\mathbf{u}) \gamma_{\alpha 3}(\mathbf{v})+a_{33 \tau 3} \gamma_{\tau 3}(\mathbf{u}) \gamma_{33}(\mathbf{v})\right]+a_{3333} \gamma_{33}(\mathbf{u}) \gamma_{33}(\mathbf{v})\right\} d x
\end{aligned}
$$


and

$$
N(e)(\mathbf{u}, \mathbf{v})=\int_{\Omega} \rho\left[u_{\alpha} v_{\alpha}+e^{2} u_{3} v_{3}\right] d x
$$

These expressions for $B(e)$ and $N(e)$ are obtained by substituting the above scalings into (9) and (10) and then factoring out $e^{6}$ so that the term in (21) with coefficient $a_{3333}$ will be multiplied by $e^{0}$.

We now prove some lemmas which will allow us to conclude that the solutions of (20) converge to the solutions of a one-dimensional eigenvalue problem as $e \rightarrow 0$. We begin by establishing a priori bounds on the eigenvalues and eigenfunctions.

LEMMA 2.1. For each integer $l \geq 1$, there exists a constant $\delta_{l}$ independent of $e$ with $\Lambda^{e, l} \leq \delta_{l}$.

Proof. For $\mathbf{v} \in \mathbf{V}$, define

$$
J(e)(\mathbf{v})=\frac{B(e)(\mathbf{v}, \mathbf{v})}{N(e)(\mathbf{v}, \mathbf{v})}
$$

Then $\Lambda^{l}(e)$ is given by

$$
\Lambda^{l}(e)=\min _{U \in \mathscr{V}_{l}}\left\{\max _{\mathbf{v} \in U} J(e)(\mathbf{v})\right\}
$$

Let $\mathscr{S}_{l}$ denote the family of all $l$-dimensional subspaces of $H_{L}^{2}(0, L)$ where

$$
H_{L}^{2}(0, L)=\left\{\varphi \in H^{2}(0, L) \mid \varphi(0)=\varphi^{\prime}(0)=0\right\},
$$

and let $U_{S}=\left\{\mathbf{v}_{\varphi}=\left(\varphi\left(x_{3}\right), 0,-x_{1} \varphi^{\prime}\left(x_{3}\right)\right) \mid \varphi \in S\right\}$ for each $S \in \mathscr{S}_{l}$. Then $\left\{U_{S} \mid S \in \mathscr{S}_{l}\right\} \subset \mathscr{V}_{l}$; so

$$
\begin{aligned}
\Lambda^{l}(e) & \leq \min _{S \in \mathscr{S}_{l}}\left\{\max _{\mathbf{v}_{\varphi} \in U_{S}} J(e)\left(\mathbf{v}_{\varphi}\right)\right\} \\
& =\min _{S \in \mathscr{S}_{l}}\left\{\max _{\varphi \in S} \frac{\int_{\Omega} a_{3333}\left[x_{1} \varphi^{\prime \prime}\left(x_{3}\right)\right]^{2} d x}{\rho \int_{\Omega}\left[\varphi^{2}\left(x_{3}\right)+e^{2} x_{1}^{2}\left(\varphi^{\prime}\left(x_{3}\right)\right)^{2}\right] d x}\right\} \\
& \leq \min _{S \in \mathscr{S}_{l}}\left\{\max _{\varphi \in S} r(\varphi)\right\}=\delta_{l},
\end{aligned}
$$

where

$$
r(\varphi)=\frac{\int_{0}^{L}\left(\int_{S\left(x_{3}\right)} x_{1}^{2} d x_{1} d x_{2}\right) a_{3333}\left(\varphi^{\prime \prime}\left(x_{3}\right)\right)^{2} d x_{3}}{\rho \int_{0}^{L}\left|S\left(x_{3}\right)\right| \varphi^{2}\left(x_{3}\right) d x_{3}} .
$$

Note: Taking $\mathbf{v}=\left(v_{i j}\right)$ with $v_{33}=1$, all other $v_{i j}=0$ in (4)(ii) yields $\alpha_{0} \leq$ $a_{3333}$.

LEMMA 2.2. For each integer $l \geq 1$, there exists a constant $C_{l}$ independent of $e$ such that

$$
\left\|\mathbf{u}_{l}(e)\right\|_{1, \Omega} \leq C_{l}
$$

and

$$
\left|\gamma_{\alpha \beta}\left(\mathbf{u}_{l}(e)\right)\right|_{0, \Omega} \leq C_{l} e^{2}, \quad\left|\gamma_{\alpha 3}\left(\mathbf{u}_{l}(e)\right)\right|_{0, \Omega} \leq C_{l} e, \quad\left|\gamma_{33}\left(\mathbf{u}_{l}(e)\right)\right|_{0, \Omega} \leq C_{l}
$$


Hence, there exists a subsequence of $\left\{\left(\Lambda^{l}(e), \mathbf{u}_{l}(e)\right)\right\}$, which we still denote by $\left\{\left(\Lambda^{l}(e), \mathbf{u}_{l}(e)\right)\right\}$, such that

$$
\Lambda^{l}(e) \rightarrow \Lambda^{l}, \quad \mathbf{u}_{l}(e) \rightarrow \mathbf{u}_{l}^{*} \quad \text { weakly in } \mathbf{V} ;
$$

so $\mathbf{u}_{l}^{*}=0$ on $\Gamma_{0}$ and

$$
\gamma_{\alpha j}\left(\mathbf{u}_{l}^{*}\right)=0 \text {. }
$$

Furthermore, we can use the same subsequence for all $l>1$.

Proof. Take $\mathbf{u}(e)=\mathbf{v}=\mathbf{u}_{l}(e)$ in (20). Then, by (13), we get

$$
B(e)\left(\mathbf{u}_{l}(e), \mathbf{u}_{l}(e)\right)=\Lambda_{l}(e) .
$$

Set $\tilde{\gamma}_{\alpha \beta}^{e}=e^{-2} \gamma_{\alpha \beta}\left(\mathbf{u}_{l}(e)\right), \tilde{\gamma}_{\alpha 3}^{e}=e^{-1} \gamma_{\alpha 3}\left(\mathbf{u}_{l}(e)\right)$, and $\tilde{\gamma}_{33}^{e}=\gamma_{33}\left(\mathbf{u}_{l}(e)\right)$. If we assume, without loss of generality, that $e \leq 1$, then by the assumptions (4) on the $a_{i j k h}$,

$$
\begin{gathered}
\alpha_{0} \int_{\Omega} \gamma_{i j}\left(\mathbf{u}_{l}(e)\right) \gamma_{i j}\left(\mathbf{u}_{l}(e)\right) d x \\
\leq \alpha_{0} \int_{\Omega} \tilde{\gamma}_{i j}^{e} \tilde{\gamma}_{i j}^{e} d x \\
\leq \int_{\Omega} a_{i j k h} \tilde{\gamma}_{k h}^{e} \tilde{\gamma}_{i j}^{e} d x \\
\quad=B(e)\left(\mathbf{u}_{l}(e), \mathbf{u}_{l}(e)\right) .
\end{gathered}
$$

Using Theorem 6.3-4 in [2] (which is a consequence of Korn's Inequality) and Lemma 2.1 , the desired bounds follow. Hence, we can extract a weakly convergent subsequence, and, by a diagonalization process, the same subsequence works for all $l$.

Remark. In [8] Cioranescu and Saint Jean Paulin obtain the result

$$
\|\mathbf{u}\|_{1, \Omega} \leq C e^{-3}
$$

where $\mathbf{u}$ is the solution of the scaled problem. Recall that in this paper we are considering the problem of free vibration. In [8], however, the truss is subjected to body forces $\mathbf{F}^{e}$ and surface forces $\mathbf{G}^{e}$ where $F_{\alpha}^{e}=O(1), F_{3}^{e}=O\left(e^{-1}\right), G_{\alpha}^{e}=O(e)$, and $G_{3}^{e}=O(1)$, or, in terms of the scaled forces, $F_{\alpha}=O\left(e^{-1}\right), F_{3}=O\left(e^{-3}\right)$, $G_{\alpha}=O(1)$, and $G_{3}=O\left(e^{-2}\right)$. Hence the difference in the estimates on the scaled displacements. We remark also that this difference in the assumptions on the applied forces leads us to different choices for the test functions used below to obtain the limits of the moments.

Our next goal is to discover the form of the function $\mathbf{u}_{l}^{*}$. Since the argument is exactly the same as in the static case (see [8]), we only summarize the results. Using (24) we get

$$
u_{l, \alpha}^{*}(\mathbf{x})=V_{l, \alpha}^{*}\left(x_{3}\right) \in H_{L}^{1}(0, L),
$$

and

$$
u_{l, 3}^{*}(\mathbf{x})=-x_{1} \frac{d V_{l, 1}^{*}\left(x_{3}\right)}{d x_{3}}-x_{2} \frac{d V_{l, 2}^{*}\left(x_{3}\right)}{d x_{3}}+V_{l, 3}^{*}\left(x_{3}\right),
$$


where $V_{l, 3}^{*} \in H_{L}^{1}(0, L)$. We want to argue that $V_{l, \alpha}^{*} \in H_{L}^{2}(0, L)$. We can identify $V_{l, 3}^{*}$ with a function in $\mathbf{V}$. Thus by (26),

$$
\int_{\Omega}\left(x_{\alpha} \frac{d^{2} V_{l, \alpha}^{*}\left(x_{3}\right)}{d x_{3}^{2}}\right)^{2} d x=\int_{\Omega}\left(\frac{\partial V_{l, 3}^{*}}{\partial x_{3}}-\frac{\partial u_{l, 3}^{*}}{\partial x_{3}}\right)^{2} d x \leq C .
$$

But, by (1),

$$
\begin{aligned}
\int_{\Omega}\left(x_{\alpha} \frac{d^{2} V_{l, \alpha}^{*}}{d x_{3}^{2}}\right)^{2} d x & =\int_{0}^{L} \int_{S\left(x_{3}\right)}\left(x_{\alpha} \frac{d^{2} V_{l, \alpha}^{*}}{d x_{3}^{2}}\right)^{2} d x_{1} d x_{2} d x_{3} \\
& =\int_{0}^{L}\left(\int_{S\left(x_{3}\right)} x_{\alpha}^{2} d x_{1} d x_{2}\right)\left(\frac{d^{2} V_{l, \alpha}^{*}}{d x_{3}^{2}}\right)^{2} d x_{3}
\end{aligned}
$$

Hence $V_{l, \alpha}^{*} \in H^{2}(0, L)$, and by $(26)$,

$$
x_{\alpha} \frac{d V_{l, \alpha}^{*}}{d x_{3}}(0)=V_{l, 3}^{*}(0)-u_{l, 3}^{*}\left(x_{1}, x_{2}, 0\right)
$$

for all $\left(x_{1}, x_{2}\right) \in \Gamma$ which implies that $d V_{l, \alpha}^{*}(0) / d x_{3}=0$.

Next, we want to derive the eigenvalue problem satisfied by $\left(\Lambda_{l}, \mathbf{u}_{l}^{*}\right)$. Again, the arguments are very similar to those given in [8] for the static case. We define

$$
\sigma_{i j}^{l}(e)=a_{i j \alpha \beta} \gamma_{\alpha \beta}\left(\mathbf{u}_{l}(e)\right)+2 e a_{i j 3 \beta} \gamma_{3 \beta}\left(\mathbf{u}_{l}(e)\right)+e^{2} a_{i j 33} \gamma_{33}\left(\mathbf{u}_{l}(e)\right) .
$$

Then by the a priori estimates of Lemma 2.2 ,

$$
\left|\sigma_{i j}^{l}(e)\right|_{0, \Omega} \leq C_{l} e^{2}
$$

which implies the existence of a subsequence such that

$$
e^{-2} \sigma_{i j}^{l}(e) \rightarrow \sigma_{l, i j}^{*} \quad \text { weakly in } L^{2}(\Omega)
$$

Define

$$
M_{l, \alpha}^{e}\left(x_{3}\right)=\int_{S\left(x_{3}\right)} x_{\alpha} \sigma_{33}^{l}(e) d x_{1} d x_{2}
$$

Then, by (27), $e^{-2} M_{l, \alpha}^{e} \rightarrow M_{l, \alpha}^{*}$ weakly in $L^{2}(0, L)$. Define

$$
\mathscr{V}=\left\{\psi \in C^{\infty}(0, L) \mid \psi^{(n)}(0)=0 \text { for all } n\right\} .
$$

Take $\mathbf{u}(e)=\mathbf{u}_{l}(e)$ and $\mathbf{v}=\left(\varphi\left(x_{3}\right), 0,-x_{1} \varphi^{\prime}\left(x_{3}\right)\right)$ for $\varphi \in \mathscr{V}$ in (20). Observe that $\gamma_{\alpha \beta}(\mathbf{v})=\gamma_{\alpha 3}(\mathbf{v})=0$ for all choices of $\alpha$ and $\beta$. Thus, using (28) we get

$$
-\int_{0}^{L} e^{-2} M_{l, 1}^{e}\left(x_{3}\right) \varphi^{\prime \prime}\left(x_{3}\right) d x_{3}=\Lambda_{l}(e) \int_{\Omega} \rho\left[u_{l, 1}(e) \varphi\left(x_{3}\right)-e^{2} u_{l, 3}(e) x_{1} \varphi^{\prime}\left(x_{3}\right)\right] d x .
$$

Letting $e \rightarrow 0$ we obtain

$$
-\int_{0}^{L} M_{l, 1}^{*} \varphi^{\prime \prime} d x_{3}=\rho \Lambda_{l} \int_{0}^{L}\left|S\left(x_{3}\right)\right| V_{l, 1}^{*}\left(x_{3}\right) \varphi\left(x_{3}\right) d x_{3} .
$$


Upon integrating by parts we obtain

$$
-M_{l, 1}^{*}(L) \varphi^{\prime}(L)+\frac{d M_{l, 1}^{*}}{d x_{3}}(L) \varphi(L)-\int_{0}^{L} \frac{d^{2} M_{l, 1}^{*}}{d x_{3}^{2}} \varphi d x_{3}=\rho \Lambda_{l} \int_{0}^{L}\left|S\left(x_{3}\right)\right| V_{l, 1}^{*} \varphi d x_{3}
$$

for all $\varphi \in \mathscr{V}$. It is clear that we can do the same calculations with $\mathbf{v}=\left(0, \varphi\left(x_{3}\right)\right.$, $\left.-x_{2} \varphi^{\prime}\left(x_{3}\right)\right)$; so $M_{l, \alpha}^{*}$ satisfies

$$
-\frac{d^{2} M_{l, \alpha}^{*}}{d x_{3}^{2}}=\rho \Lambda_{l}\left|S\left(x_{3}\right)\right| V_{l, \alpha}^{*}, \quad M_{l, \alpha}^{*}(L)=\frac{d M_{l, \alpha}^{*}}{d x_{3}}(L)=0 .
$$

Just as in [8] we obtain for the other moments

$$
\int_{S\left(x_{3}\right)} x_{\eta} \sigma_{\alpha \beta}^{*} d x_{1} d x_{2}=0
$$

and

$$
\int_{S\left(x_{3}\right)} \sigma_{\alpha \beta}^{*} d x_{1} d x_{2}=0
$$

Observe, however, that taking $\mathbf{v}=\left(0,0, \varphi\left(x_{3}\right)\right)$ with $\varphi \in \mathscr{V}$ in $(20)$ and passing to the limit, we obtain

$$
\int_{S\left(x_{3}\right)} \sigma_{33}^{*} d x_{1} d x_{2}=0
$$

This result is in contrast to the corresponding result in the static case. As mentioned above, the reason for this difference is that our truss is not subjected to any external forces.

Next we need the following result which is proved in [8]:

LemMa 2.3. The tensor $\left(a_{\beta j \tau i}\right)$ is positive definite, hence has an inverse $\left(b_{\beta j \tau i}\right)$. Using this inverse tensor, $\sigma_{l, 33}^{*}$ has the form

$$
\sigma_{l, 33}^{*}=a_{33 \tau i} b_{\beta j \tau i} \sigma_{l, \beta j}^{*}+\left(a_{3333}-a_{33 \tau i} b_{\beta j \tau i} a_{\beta j 33}\right) \gamma_{33}\left(\mathbf{u}_{l}^{*}\right) .
$$

Using (27), (28), and (33) we get

$$
\begin{aligned}
M_{l, \alpha}^{*}= & \int_{S\left(x_{3}\right)} x_{\alpha} \sigma_{l, 33}^{*} d x_{1} d x_{2} \\
= & a_{33 \tau i} b_{\beta j \tau i} \int_{S\left(x_{3}\right)} x_{\alpha} \sigma_{l, \beta j}^{*} d x_{1} d x_{2} \\
& +\left(a_{3333}-a_{33 \tau i} b_{\beta j \tau i} a_{\beta j 33}\right) \int_{S\left(x_{3}\right)} x_{\alpha} \gamma_{33}\left(\mathbf{u}_{l}^{*}\right) d x_{1} d x_{2} .
\end{aligned}
$$

By (30), this reduces to

$$
\begin{aligned}
M_{l, \alpha}^{*}= & a_{33 \tau i} b_{\beta 3 \tau i} \int_{S\left(x_{3}\right)} x_{\alpha} \sigma_{l, \beta 3}^{*} d x_{1} d x_{2} \\
& +\left(a_{3333}-a_{33 \tau i} b_{\beta j \tau i} a_{\beta j 33}\right) \int_{S\left(x_{3}\right)} x_{\alpha} \gamma_{33}\left(\mathbf{u}_{l}^{*}\right) d x_{1} d x_{2} .
\end{aligned}
$$


All the results obtained thus far hold for any elasticity coefficients $a_{i j k h}$. We now assume that the material constituting the truss is isotropic and homogeneous, i.e., that the $a_{i j k h}$ are given by

$$
a_{i j k h}=\lambda \delta_{i j} \delta_{k h}+\mu\left(\delta_{i k} \delta_{j h}+\delta_{i h} \delta_{j k}\right),
$$

where $\lambda$ and $\mu$ are the Lamé constants. The following lemma is proved in [8]:

LEMMA 2.4. For an isotropic, homogeneous elastic material, the inverse tensor $\left(b_{\alpha j \beta i}\right)$ satisfies

$$
\begin{aligned}
& b_{1111}=b_{2222}=\frac{\lambda+2 \mu}{4 \mu(\lambda+\mu)}, \\
& b_{1122}=b_{2211}=-\frac{\lambda}{4 \mu(\lambda+\mu)}, \\
& b_{1212}=b_{2121}=\frac{1}{2 \mu}, \\
& b_{1313}=b_{2323}=\frac{1}{\mu},
\end{aligned}
$$

By (35) and (36),

all others $=0$.

$$
\begin{array}{cc}
a_{33 \tau i} b_{\beta 3 \tau i}=0 & \text { for all } \beta, \\
a_{3333}-a_{33 \tau i} b_{\beta j \tau i} a_{\beta j 33}=\frac{\mu(3 \lambda+2 \mu)}{\lambda+\mu}=E .
\end{array}
$$

Thus, (34) simplifies to

$$
M_{l, \alpha}^{*}=E \int_{S\left(x_{3}\right)} x_{\alpha} \gamma_{33}\left(\mathbf{u}_{l}^{*}\right) d x_{1} d x_{2} .
$$

Using (26) we get

$$
M_{l, \alpha}^{*}=E \int_{S\left(x_{3}\right)}\left(-x_{\alpha} x_{\beta} \frac{d^{2} V_{l, \beta}^{*}}{d x_{3}^{2}}+x_{\alpha} \frac{d V_{l, 3}^{*}}{d x_{3}}\right) d x_{1} d x_{2},
$$

but by (1),

$$
\left.M_{l, \alpha}^{*}=-E\left(\int_{S\left(x_{3}\right)} x_{\alpha}^{2} d x_{1} d x_{2}\right) \frac{d^{2} V_{l, \alpha}^{*}}{d x_{3}^{2}} \quad \text { (no summation in } \alpha\right) .
$$

Substituting (38) into (29), we get finally (no summation in $\alpha$ )

$$
\begin{gathered}
E \frac{d^{2}}{d x_{3}^{2}}\left[\left(\int_{S\left(x_{3}\right)} x_{\alpha}^{2} d x_{1} d x_{2}\right) \frac{d^{2} V_{l, \alpha}^{*}}{d x_{3}^{2}}\right]=\rho \Lambda_{l}\left|S\left(x_{3}\right)\right| V_{l, \alpha}^{*}, \\
\frac{d^{2} V_{l, \alpha}^{*}}{d x_{3}^{2}}(L)=\frac{d^{3} V_{l, \alpha}^{*}}{d x_{3}^{3}}(L)=0 .
\end{gathered}
$$

All that is left to do now is to find the equation satisfied by $V_{l, 3}^{*}$. By (31), (32), (33), and (37),

$$
0=\int_{S\left(x_{3}\right)} \sigma_{l, 33}^{*} d x_{1} d x_{2}=E \int_{S\left(x_{3}\right)} \gamma_{33}\left(\mathbf{u}_{l}^{*}\right) d x_{1} d x_{2} .
$$


Again using (26) we get

$$
E \int_{S\left(x_{3}\right)}\left(-x_{\alpha} \frac{d^{2} V_{l, \alpha}^{*}}{d x_{3}^{2}}\left(x_{3}\right)+\frac{d V_{l, 3}^{*}}{d x_{3}}\left(x_{3}\right)\right) d x_{1} d x_{2}=0
$$

and (1) implies that

$$
E\left|S\left(x_{3}\right)\right| \frac{d V_{l, 3}^{*}}{d x_{3}}\left(x_{3}\right)=0
$$

which implies that

$$
\frac{d V_{l, 3}^{*}}{d x_{3}}\left(x_{3}\right)=0
$$

In the same way that we got the boundary conditions in (29), we also get

$$
\frac{d V_{l, 3}^{*}}{d x_{3}}(L)=0
$$

This equation and boundary condition, combined with the fact that $V_{l, 3}^{*} \in H_{L}^{1}(0, L)$, imply that

$$
V_{l, 3}^{*} \equiv 0
$$

In [8] Cioranescu and Saint Jean Paulin obtained a nontrivial equation satisfied by $V_{\delta, 3}^{\varepsilon}$ (using their notation) under the assumption that the structure is subjected to applied body forces $\mathbf{F}^{e}$ with $F_{\alpha}^{e}=O(1)$, but $F_{3}^{e}=O\left(e^{-1}\right)$. Once again, we point out that the different result obtained here is due to the fact that we are treating the problem of free vibration.

We summarize the results of this section in the following theorem which covers the general case $Q^{e}=\omega^{e} \times(0, L)$.

THEOREM 2.5. Let $\Omega^{e}$ be a periodic structure made of a homogeneous, isotropic elastic material and satisfying the geometric assumption (1). Let $\left(\Lambda_{k, \alpha}, V_{k, \alpha}\right)$ denote the solutions of (39) for $\alpha=1,2$. If $(\Lambda(e), \mathbf{u}(e))$ is a solution of the scaled problem (20), then $\Lambda(e)$ converges to some $\Lambda_{k, \alpha}$ and the corresponding eigenfunction $\mathbf{u}(e)$ converges weakly in $\mathbf{V}$ to $\mathbf{u}^{*}=\left(u_{i}^{*}\right)$ where

$$
\begin{gathered}
u_{\alpha}^{*}(\mathbf{x})=V_{k, \alpha}\left(x_{3}\right) \in H_{L}^{2}(0, L), \\
u_{3}^{*}(\mathbf{x})=-x_{1} V_{k, 1}^{\prime}\left(x_{3}\right)-x_{2} V_{k, 2}^{\prime}\left(x_{3}\right),
\end{gathered}
$$

and $V_{k, \alpha}$ is the solution of (39) corresponding to $\Lambda_{l}=\Lambda_{k, \alpha}$.

Observe that if $\Lambda_{\hat{k}, 1}$ is not in the sequence $\left\{\Lambda_{k, 2}\right\}$ for some $\hat{k}$, then $V_{\hat{k}, 2} \equiv 0$.

REMARKS 1. If $S\left(x_{3}\right)=\left(-a_{1}, a_{1}\right) \times\left(-a_{2}, a_{2}\right)$ for all $x_{3}$ (i.e., the structure under consideration is a beam with rectangular cross section rather than a truss), then (1) holds, so the above analysis carries over, and equation (39) becomes (with $\alpha=1$ )

$$
E \frac{4 a_{1}^{3} a_{2}}{3} \frac{d^{4} V_{l, 1}^{*}}{d x_{3}^{4}}\left(x_{3}\right)=\rho\left(4 a_{1} a_{2}\right) \Lambda_{l} V_{l, 1}^{*}\left(x_{3}\right) \text { on } Q
$$


Using the scalings (16)-(19) to get an equation on $Q^{e}$, then multiplying by $e^{4}$ we get

$$
E \frac{4 a_{1}^{3} a_{2} e^{4}}{3} \frac{d^{4} V_{l, 1}^{*}}{d\left(x_{3}^{e}\right)^{4}}\left(x_{3}^{e}\right)=\rho^{e} 4 a_{1} a_{2} e^{2} \Lambda_{l}^{e} V_{l, 1}^{e}\left(x_{3}^{e}\right) \text { on } Q^{e} .
$$

But the beam has width $w=2 a_{2} e$ and thickness $h=2 a_{1} e$, so $I=w h^{3} / 12=$ $16 a_{1}^{3} a_{2} e^{4} / 12$, and the cross-sectional area is $A=w h=4 a_{1} a_{2} e^{2}$. Thus, the above equation is (suppressing the $e$ ):

$$
E I \frac{d^{4} V_{\alpha}}{d x^{4}}=\rho A \Lambda V_{\alpha}
$$

This is just the usual Euler-Bernoulli beam equation. Similar calculations can be used for other structures with a uniform cross section.

2. An analysis of our results to this point leads us to certain expectations regarding the modes of vibration:

(a) If $a_{1}=a_{2}$ then $V_{l, 1}^{*}$ and $V_{l, 2}^{*}$ satisfy the same equation, so the bending modes should occur in pairs. This makes sense physically since the truss "looks the same" when rotated through an angle of $90^{\circ}$ about the $x_{3}$ axis.

(b) The form of $u_{l, \alpha}^{*}$ given by (25), i.e., the fact that $u_{l, \alpha}^{*}$ depends only on $x_{3}$, implies that our approximate model will not detect any torsional modes of vibration. What this means is that the torsional modes of the actual truss are insignificant compared to the bending modes.

(c) Since $V_{l, 3}^{*}=0$ the axial deformation is due only to bending. Thus we expect that the axial modes of vibration are also insignificant.

All these observations are in agreement with experimental data.

3. The homogenized equation. In the previous section we derived the one-dimensional eigenvalue problem

$$
\begin{array}{r}
E \frac{d^{2}}{d x_{3}^{2}}\left[\left(\int_{S\left(x_{3}\right)} x_{\alpha}^{2} d x_{1} d x_{2}\right) \frac{d^{2} V_{l, \alpha}^{*}}{d x_{3}^{2}}\right]=\rho \Lambda_{l, \alpha}\left|S\left(x_{3}\right)\right| V_{l, \alpha}^{*}, \\
V_{l, \alpha}^{*}(0)=0, \quad \frac{d V_{l, \alpha}^{*}}{d x_{3}}(0)=0, \quad \frac{d^{2} V_{l, \alpha}^{*}}{d x_{3}^{2}}(L)=0, \quad \frac{d^{3} V_{l, \alpha}^{*}}{d x_{3}^{3}}(L)=0 .
\end{array}
$$

(There is no summation in $\alpha$.) Let us now restore the $\varepsilon$, and $\delta$ which we dropped earlier. Define $y=x_{3} / \varepsilon, \Sigma_{\delta}(y)=S_{\varepsilon \delta}(\varepsilon y)$, and

$$
a_{\alpha}^{\delta}(y)=\int_{\Sigma_{\delta}(y)} x_{\alpha}^{2} d x_{1} d x_{2} .
$$

If we drop the $\alpha$ and the subscript on $x_{3}$ for simplicity, we can rewrite (41) as

$$
E \frac{d^{2}}{d x^{2}}\left(a_{\delta}\left(\frac{x}{\varepsilon}\right) \frac{d^{2} V_{l}^{\varepsilon \delta}}{d x^{2}}(x)\right)=\rho \Lambda_{l}^{\varepsilon \delta}\left|\Sigma_{\delta}(x / \varepsilon)\right| V_{l}^{\varepsilon \delta}(x),
$$

plus appropriate boundary conditions. Since the coefficients appearing in equation (42) are periodic functions of $\varepsilon$, we can use homogenization techniques (see [1, 6, $7,10,11,13,14])$ to approximate the solutions of $(42)$. 
3.1. Derivation of the homogenized equation. Using a result of Kesavan (see [10]), we can show that the eigenvalues and eigenfunctions are bounded independently of $\varepsilon$; hence we can extract a subsequence (of $\varepsilon$ ) such that for all $l \geq 1$

$$
\Lambda_{l}^{\varepsilon \delta} \rightarrow \Lambda_{l}^{\delta}
$$

and

where

$$
V_{l}^{\varepsilon \delta}-V_{l}^{\delta} \quad \text { weakly in } H_{L}^{2}(0, L)
$$

$$
E q_{\delta} \frac{d^{4} V_{l}^{\delta}}{d x^{4}}=\rho \Lambda_{l}^{\delta} \tilde{q}_{\delta} V_{l}^{\delta}
$$

plus boundary conditions. The coefficients $q_{\delta}$ and $\tilde{q}_{\delta}$ come from homogenizing the operators on the left- and right-hand sides respectively of equation (42). Since $V_{l}^{\varepsilon \delta} \rightarrow V_{l}^{\delta}$ strongly in $L^{2}(0, L), \tilde{q}_{\delta}$ is simply the average of the coefficient $\left|\Sigma_{\delta}(y)\right|$ over one period; i.e.,

$$
\tilde{q}_{\delta}=c^{-1} \int_{0}^{c}\left|\Sigma_{\delta}(y)\right| d y .
$$

The homogenization of the operator on the left-hand side is exactly the same as in the static case (see [8]); hence, we only outline the derivation here. First we define $\xi_{\delta}^{\varepsilon}=a_{\delta}(x / \varepsilon)\left(d^{2} V_{\delta}^{\varepsilon} / d x^{2}\right)$ and show that $\xi_{\delta}^{\varepsilon}-\xi_{\delta}^{*}$ weakly in $L^{2}(0, L)$. Next we consider the adjoint system

$$
\begin{gathered}
\frac{d^{2}}{d y^{2}}\left(a_{\delta}(y) \frac{d^{2} w_{\delta}}{d y^{2}}(y)\right)=0 \text { in }(0, c), \\
w_{\delta}(y)-y^{2} / 2 \text { is periodic in }(0, c) .
\end{gathered}
$$

The function $w_{\delta}(y)$ is unique up to an additive constant whose value is immaterial. Now set $\chi_{\delta}(y)=w_{\delta}(y)-y^{2} / 2$ and define

$$
\chi_{\delta}^{\varepsilon}(x)=\varepsilon^{2} \chi_{\delta}(x / \varepsilon) \text { for } x \in(0, c) .
$$

Since $\chi_{\delta}^{\varepsilon}$ is periodic, we can extend it to all of $(0, L)$. Now set $w_{\delta}^{\varepsilon}(x)=\chi_{\delta}^{\varepsilon}(x)+$ $x^{2} / 2$. We can show that $\left|w_{\delta}^{\varepsilon}\right|_{2,(0, L)}$ is bounded independently of $\varepsilon$; hence

$$
w_{\delta}^{\varepsilon}-w_{\delta}^{*} \quad \text { weakly in } H^{2}(0, L) .
$$

By the periodicity of $\chi_{\delta}^{\varepsilon}$, we next show that

$$
\frac{d w_{\delta}^{*}}{d x}=x \quad \text { and } \quad \frac{d^{2} w_{\delta}^{*}}{d x^{2}}=1
$$

Now define

$$
\eta_{\delta}(y)=a_{\delta}(y) \frac{d^{2} w_{\delta}(y)}{d y^{2}}
$$

(which is periodic in $(0, c)$ ), and $\eta_{\delta}^{\varepsilon}(x)=\eta_{\delta}(x / \varepsilon)$. From the adjoint system (43) we have

$$
\frac{d^{2} \eta_{\delta}^{\varepsilon}}{d x^{2}}=0
$$


and by the periodicity of $\eta_{\delta}$

$$
\eta_{\delta}^{\varepsilon}-c^{-1} \int_{0}^{c} a_{\delta}(y) \frac{d^{2} w_{\delta}(y)}{d y^{2}} d y \quad \text { weakly }{ }^{*} \text { in } L^{\infty}(0, L) .
$$

Suppose the static equation is

$$
E \frac{d^{2}}{d x^{2}}\left(a_{\delta}\left(\frac{x}{\varepsilon}\right) \frac{d^{2} V_{\delta}^{\varepsilon}}{d x^{2}}\right)=f^{\varepsilon}
$$

Then $\xi_{\delta}^{\varepsilon}$ satisfies

$$
E \frac{d^{2} \xi_{\delta}^{\varepsilon}}{d x^{2}}=f^{\varepsilon}
$$

Let $\varphi \in \mathscr{D}(0, L)$, multiply (44) by $\varphi V_{\delta}^{\varepsilon}$ and (46) by $\varphi w_{\delta}^{\varepsilon}$, subtract, integrate by parts, and pass to the limit. We get the desired result where $q_{\delta}$ is the weak ${ }^{*}$ limit of $\eta_{\delta}^{\varepsilon}$ given in (45).

3.2. Explicit computation of the homogenized coefficients. We will calculate the coefficients for several cases of the representative period, but first we express $q_{\delta}$ in terms of $a_{\delta}$. Integrating (43) twice we get

$$
a_{\delta}(y)=\frac{d^{2} w_{\delta}}{d y^{2}}(y)=c_{1} y+c_{2} .
$$

By the periodicity of $a_{\delta}$ and of the second derivative of $w_{\delta}(y)-y^{2} / 2$ we get $c_{1}=0$. Hence, $d^{2} w_{\delta} / d y^{2}(y)=c_{2}\left(a_{\delta}(y)\right)^{-1}$, which implies

$$
q_{\delta}=c^{-1} \int_{0}^{c} a_{\delta}(y) \frac{d^{2} w_{\delta}}{d y^{2}}(y) d y=c_{2}
$$

Integrating again we get

$$
\frac{d w_{\delta}}{d y}(y)=q_{\delta} \int_{0}^{y}\left(a_{\delta}(\eta)\right)^{-1} d \eta+c_{3} .
$$

By the periodicity of $\frac{d w_{\delta}}{d y}(y)-y$ we have

$$
c_{3}=q_{\delta} \int_{0}^{c}\left(a_{\delta}(y)\right)^{-1} d y+c_{3}-c
$$

which implies

$$
\left(q_{\delta}\right)^{-1}=c^{-1} \int_{0}^{c}\left(a_{\delta}(y)\right)^{-1} d y .
$$

3.2.1. Trusses with square cross section. We will consider four cases for the representative period. In each case the truss will be assumed to have a square cross section (so that $\omega=\left(-a_{1}, a_{1}\right) \times\left(-a_{2}, a_{2}\right)$ with $\left.a_{1}=a_{2}\right)$, and all bars comprising the truss will have the same cross section. Since the truss is square, we have $a_{1}^{\delta}(y)=a_{2}^{\delta}(y)$ for all $y$, so for the sake of definiteness, we take $a_{\delta}=a_{1}^{\delta}$ for all calculations. In each case for which we compute the coefficients, we will see that $q_{\delta}=O\left(\delta^{2}\right)$ and 
$\tilde{q}_{\delta}=O\left(\delta^{2}\right)$. Hence, using the same type of arguments as before, we can extract a weakly convergent subsequence of eigenfunctions. In each case the resulting equation in final form (unscaled) will be

$$
q^{*} E I V^{\prime \prime \prime \prime}=\rho A \omega^{2} V .
$$

Case 1. We first consider the case in which the representative period is a cube with all the material along the edges (i.e., no oblique bars). Thus, we set $a_{1}=a_{2}=1$ and $c=2$ and take the cross section of the bars to be $\delta \times \delta$. (We could just as easily have taken the cross section to be $2 \delta \times 2 \delta$, or $a_{1}=a_{2}=1 / 2, c=1$ with cross section $\delta \times \delta$. Regardless of the choice we get the same final result.) By straightforward calculations we see that

$$
\left|\Sigma_{\delta}(y)\right|= \begin{cases}4 \delta(2-\delta), & y \in(0, \delta / 2) \cup(2-\delta / 2,2), \\ 4 \delta^{2}, & y \in(\delta / 2,2-\delta / 2)\end{cases}
$$

Hence,

$$
\begin{aligned}
\delta^{-2} \tilde{q}_{\delta} & =\left(2 \delta^{2}\right)^{-1} \int_{0}^{2}\left|\Sigma_{\delta}(y)\right| d y \\
& =\left(2 \delta^{2}\right)^{-1}\left[4 \delta^{2}(2-\delta)+4 \delta(2-\delta) \delta\right]=4(2-\delta) \rightarrow 8
\end{aligned}
$$

as $\delta \rightarrow 0$. Next, for $y \in(0, \delta / 2) \cup(2-\delta / 2,2)$,

$$
a_{\delta}(y)=\int_{-1}^{1} \int_{-1}^{1} x_{1}^{2} d x_{1} d x_{2}-\int_{-1+\delta}^{1-\delta} \int_{-1+\delta}^{1-\delta} x_{1}^{2} d x_{1} d x_{2}=4 \delta\left(4-6 \delta+4 \delta^{2}-\delta^{3}\right) / 3,
$$

and for $\delta / 2<y<2-\delta / 2$,

$$
a_{\delta}(y)=4 \int_{1-\delta}^{1} \int_{1-\delta}^{1} x_{1}^{2} d x_{1} d x_{2}=4 \delta^{2}\left(3-3 \delta+\delta^{2}\right) / 3 .
$$

Thus,

which implies

$$
q_{\delta}^{-1}=\frac{1}{2}\left[\frac{3 \delta}{4 \delta\left(4-6 \delta+4 \delta^{2}-\delta^{3}\right)}+\frac{3(2-\delta)}{4 \delta^{2}\left(3-3 \delta+\delta^{2}\right)}\right],
$$

$$
\delta^{2} q_{\delta}^{-1}=\frac{1}{2}\left[\frac{3 \delta^{2}}{4\left(4-6 \delta+4 \delta^{2}-\delta^{3}\right)}+\frac{3(2-\delta)}{4\left(3-3 \delta+\delta^{2}\right)}\right] \rightarrow \frac{1}{4} \quad \text { as } \delta \rightarrow 0 .
$$

Hence, $\delta^{-2} q_{\delta} \rightarrow 4$ as $\delta \rightarrow 0$, and the limit equation is

$$
4 E V^{\prime \prime \prime \prime}=8 \rho \Lambda V \text {. }
$$

Recalling the scaling of the density (equation (19)), multiplying by $e^{4}$, and writing $\omega^{2}$ for $\Lambda$, this equation becomes

$$
4 e^{4} E V^{\prime \prime \prime \prime}=8 e^{2} \rho \omega^{2} V .
$$

Since the width of the truss is $w=2 e$, and the thickness is $h=2 e$, we get $I=$ $\int_{w^{e}}\left(x_{1}^{e}\right)^{2} d x_{1}^{e} d x_{2}^{e}=w h^{3} / 12=4 e^{4} / 3$ and $A=w h=4 e^{2}$. Thus,

$$
\frac{3}{2} E I V^{\prime \prime \prime \prime}=\rho A \omega^{2} V \text {, }
$$

where $\rho$ now represents the unscaled density. 
Case 2. We now take as the representative period a cube with two oblique cross bars on each vertical face (i.e., the first structure in Fig. 3). Again we take $a_{1}=a_{2}=1$ and $c=2$, and all the bars in the truss have cross section $\delta \times \delta$. Now $\Sigma_{\delta}(y)$ is as shown in Fig. 5 where $\eta=y-1+\sqrt{2} \delta / 2$, and $\tilde{\eta}=\eta-\sqrt{2} \delta$. Thus,

$$
\left|\Sigma_{\delta}(y)\right| \approx \begin{cases}4 \delta(2-\delta), & y \in(0, \delta / 2) \cup(2-\delta / 2,2), \\ 4 \delta^{2}+8 \sqrt{2} \delta^{2}, & y \in(\delta / 2,2-\delta / 2) ;\end{cases}
$$

hence, $\tilde{q}_{\delta} \approx 2 \delta^{2}(2-\delta)+(2-\delta)(2+4 \sqrt{2}) \delta^{2}$, and so $\delta^{-2} \tilde{q}_{\delta} \rightarrow 8(1+\sqrt{2})$ as $\delta \rightarrow 0$. The above formula is only approximate because the actual area is less when the oblique bars intersect either the horizontal bars or each other. Nevertheless, the leading term is not affected, and the limit is correct.

The only difference in $a_{\delta}(y)$ from the example in Case 1 is the contribution for the oblique bars which is (since $x_{1}^{2}$ is an even function):

$$
\begin{gathered}
4\left[\int_{1-\delta}^{1} \int_{\eta-\sqrt{2} \delta}^{\eta} x_{1}^{2} d x_{1} d x_{2}+\int_{\eta-\sqrt{2} \delta}^{\eta} \int_{1-\delta}^{1} x_{1}^{2} d x_{1} d x_{2}\right] \\
=4 \sqrt{2} \delta^{2}\left(y^{2}-2 y+2-\delta+\delta^{2} / 2\right) .
\end{gathered}
$$

Thus, for $y \in(\delta / 2,2-\delta / 2)$,

$$
a_{\delta}(y) \approx 4 \delta^{2}\left(3-3 \delta+\delta^{2}\right) / 3+4 \sqrt{2} \delta^{2}\left(y^{2}-y+2-\delta+\delta^{2} / 2\right),
$$

and so,

$$
\begin{aligned}
\delta^{2}\left(q_{\delta}\right)^{-1} & =\frac{1}{2} \int_{0}^{2} \delta^{2}\left(a_{\delta}(y)\right)^{-1} d y \\
& \approx \frac{1}{2}\left[\frac{3 \delta^{2}}{4\left(4-6 \delta+4 \delta^{2}-\delta^{3}\right)}\right. \\
& \left.\quad+\int_{\delta / 2}^{2-\delta / 2} \frac{d y}{4\left(3-3 \delta+\delta^{2}\right) / 3+4 \sqrt{2}\left(y^{2}-2 y+2-\delta+\delta^{2} / 2\right)}\right],
\end{aligned}
$$

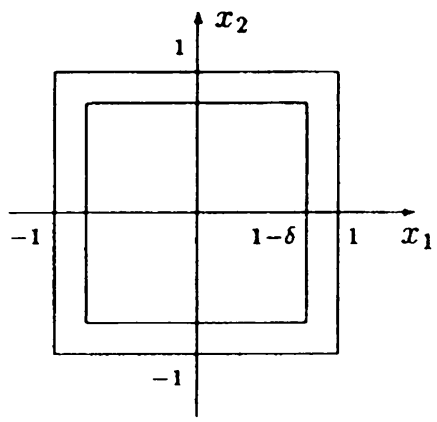

(a) $y \in\left(0, \frac{\delta}{2}\right) \cup\left(2-\frac{\delta}{2}, 2\right)$

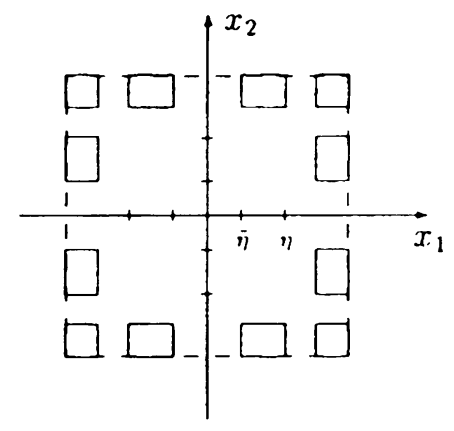

(b) $\frac{\delta}{2}<y<2-\frac{\delta}{2}$

Fig. 5. The set $\Sigma_{\delta}(y)$ for Case 2 
which implies that

$$
\begin{aligned}
\delta^{2} q_{\delta}^{-1} & \rightarrow \frac{1}{2} \int_{0}^{2} \frac{d y}{4+4 \sqrt{2}\left(y^{2}-2 y+2\right)} \\
& =\frac{1}{8 \sqrt{2}} \int_{-1}^{1} \frac{d z}{z^{2}+(2+\sqrt{2}) / 2}=\frac{1}{4} \sqrt{\frac{2-\sqrt{2}}{2}} \arctan \sqrt{2-\sqrt{2}}
\end{aligned}
$$

This time our equation is

$$
\left(\sqrt{\frac{2-\sqrt{2}}{2}} \arctan \sqrt{2-\sqrt{2}}\right)^{-1} 4 E V^{\prime \prime \prime \prime}=\rho[8(1+\sqrt{2})] \omega^{2} V,
$$

and unscaled

$$
\frac{3 \sqrt{2-\sqrt{2}}}{2 \arctan \sqrt{2-\sqrt{2}}} E I V=\rho A \omega^{2} V .
$$

In this equation, the value of the coefficient $q^{*}$ is approximately 1.7574 .

Case 3. Now we consider a truss with oblique bars in alternating directions as in the middle picture in Fig. 3. This time we take $a_{1}=a_{2}=1, c=4$ and again the cross section of the bars is $\delta \times \delta$. For $y \in(0, \delta / 2) \cup(2-\delta / 2,2+\delta / 2) \cup(4-\delta / 2,4)$, the set $\Sigma_{\delta}(y)$ is the same as in Fig. 5a. Since there are only half as many oblique bars as in the previous case, the additional contribution is only half as much. Thus,

$$
\left|\Sigma_{\delta}(y)\right| \approx \begin{cases}4 \delta(2-\delta), & y \in(0, \delta / 2) \cup(2-\delta / 2,2+\delta / 2) \cup(4-\delta / 2,4), \\ 4 \delta^{2}(1+\sqrt{2}), & y \in(\delta / 2,2-\delta / 2) \cup(2+\delta / 2,4-\delta / 2),\end{cases}
$$

and for $y \in(\delta / 2,2-\delta / 2) \cup(2+\delta / 2,4-\delta / 2)$,

$$
a_{\delta}(y) \approx 4 \delta^{2}\left(3-3 \delta+\delta^{2}\right) / 3+2 \sqrt{2} \delta^{2}\left(y^{2}-2 y+2-\delta+\delta^{2} / 2\right) .
$$

Hence,

$$
\tilde{q}_{\delta} \approx \frac{1}{4}\left[2 \delta(4 \delta)(2-\delta)+(4-2 \delta)\left(4 \delta^{2}\right)(1+\sqrt{2})\right]=2 \delta^{2}(2-\delta)(2+\sqrt{2}),
$$

so $\delta^{-2} \tilde{q}_{\delta} \rightarrow 4(2+\sqrt{2})$ as $\delta \rightarrow 0$. Also, by the same kind of analysis as in Case 2, we find that

$$
\delta^{-2} q_{\delta} \rightarrow \frac{2 \sqrt{2 \sqrt{2}+2}}{\arctan \sqrt{\sqrt{2}-1}} .
$$

Thus, in this case the (unscaled) equation is

$$
\frac{3 \sqrt{\sqrt{2}-1}}{2 \arctan \sqrt{\sqrt{2}-1}} E I V^{\prime \prime \prime \prime}=\rho A \omega^{2} V \text {. }
$$

Here $q^{*} \approx 1.6882$.

Case 4. For the final example we add a diagonal cross bar on each horizontal layer (see the third picture in Fig. 3). The only difference to $\Sigma_{\delta}(y)$ is when $y \in$ $(0, \delta / 2) \cup(2-\delta / 2,2+\delta / 2) \cup(4-\delta / 2,4)$. The top and bottom bases of the period are shown in Fig. 6, and the middle layer is the same picture rotated $90^{\circ}$. Verifying the geometric assumption in this case is not much harder than in the previous cases. 


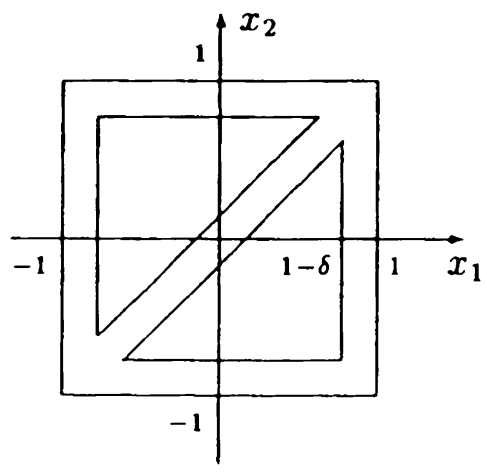

Fig. 6. The set $\Sigma_{\delta}(y)$ for $y \in(0, \delta / 2) \cup(4-\delta / 2,4)$.

For example, $\int_{\Sigma_{\delta}(y)} x_{1} d x_{1} d x_{2}=0$ since the integrand is odd in $x_{1}$ and even in $x_{2}$; so the integral below the line $x_{1}=x_{2}$ is the negative of the integral above the line. The measure of $\Sigma_{\delta}(y)$ is 4 minus the areas of the two triangles; i.e.,

$$
\left|\Sigma_{\delta}(y)\right|=(8+2 \sqrt{2}) \delta-(9 / 2+2 \sqrt{2}) \delta^{2} .
$$

After a little calculation we get $\delta^{-2} \tilde{q}_{\delta} \rightarrow 8+5 \sqrt{2}$ as $\delta \rightarrow 0$, but adding the horizontal cross bars does not affect the limit of $\delta^{-2} q_{\delta}$. The limit equation in this case is

$$
\frac{6 \sqrt{17 \sqrt{2}-23}}{7 \arctan \sqrt{\sqrt{2}-1}} E I V^{\prime \prime \prime \prime}=\rho A \omega^{2} V
$$

and $q^{*} \approx 1.5298$.

EXAMPLE. Consider a steel truss having cross section $1 \mathrm{ft} \times 1 \mathrm{ft}$ and length $10 \mathrm{ft}$. We use the values $\rho=.283 \mathrm{lb} / \mathrm{in}^{3}=.283 / 386.4 \mathrm{slug} / \mathrm{in}^{3}$ (observe that the handbook value of $\rho$ is in the units weight/volume, so we must divide by the gravitational acceleration in order to make the units mass/volume) and $E=29 \times 10^{6} \mathrm{lb} / \mathrm{in}^{2}$. Also, $A=144 \mathrm{in}^{2}$ and $I=1728 \mathrm{in}^{4}$. If we define

$$
\lambda^{4}=\frac{\rho A \omega^{2} L^{4}}{q^{*} E I},
$$

then the circular frequencies $\omega$ are given by

$$
\omega=\sqrt{\frac{q^{*} E I}{\rho A}}\left(\frac{\lambda}{L}\right)^{2}
$$

and $\lambda$ are the solutions of the frequency equation $1+\cos \lambda \cosh \lambda=0$. The first three values of $\lambda$ are $1.875,4.694$, and 7.855 , and $\lambda \sim\left(n-\frac{1}{2}\right) \pi$. The natural frequencies of vibration are given by $\omega / 2 \pi$. Table 1 on the next page shows the first three frequencies for each of the four cases discussed above.

Observe that adding the horizontal cross bars to the truss in Case 4 essentially amounts to making the truss more massive without increasing its stiffness. Hence the frequencies are lower than in Case 3. 
TABLE 1. The first three frequencies for the four square trusses.

\begin{tabular}{|cllll|}
\hline Case & $q^{*}$ & $\omega_{1} / 2 \pi$ & $\omega_{2} / 2 \pi$ & $\omega_{3} / 2 \pi$ \\
\hline 1 & 1.5 & 32.807 & 205.56 & 575.71 \\
2 & 1.7574 & 35.511 & 222.47 & 623.15 \\
3 & 1.6882 & 34.804 & 218.07 & 610.75 \\
4 & 1.5298 & 33.131 & 207.59 & 581.39 \\
\hline
\end{tabular}

3.2.2. A truss with triangular cross section. As a final example we consider a truss whose cross section is an equilateral triangle, each of whose sides has length $2 e$ with one side parallel to the $x_{1}^{e}$-axis. We take $\omega$ to be the region bounded by the triangle with vertices at $( \pm 1,-\sqrt{3} / 3)$ and $(0,2 \sqrt{3} / 3)$. We take $c=2$ and assume that the horizontal bars of the truss have cross section $\delta \times \delta$. Thus, for $y \in(0, \delta / 2) \cup$ $(2-\delta / 2,2)$, the set $\Sigma_{\delta}(y)$ is formed by removing from $\omega$ the region bounded by the triangle with vertices at $(-1+\sqrt{3} \delta,-\sqrt{3} / 3+\delta),(1-\sqrt{3} \delta,-\sqrt{3} / 3+\delta)$, and $(0,2 \sqrt{3} / 3-2 \delta)$. We assume that the vertical bars of the truss have triangular cross section with each side of length $4 \sqrt{3} \delta / 3$ (i.e., an equilateral triangle of altitude $2 \delta$ ). It is clear that

$$
\left|\Sigma_{\delta}(y)\right|= \begin{cases}3 \delta(2-\sqrt{3} \delta), & y \in(0, \delta / 2) \cup(2-\delta / 2,2), \\ 4 \sqrt{3} \delta^{2}, & y \in(\delta / 2,2-\delta / 2) .\end{cases}
$$

Thus, $\tilde{q}_{\delta}=\frac{1}{2}\left[3 \delta^{2}(2-\sqrt{3} \delta)+4 \sqrt{3} \delta^{2}(2-\delta)\right]=(3+4 \sqrt{3}) \delta^{2}-7 \sqrt{3} \delta^{3} / 2$, and so $\delta^{-2} \tilde{q}_{\delta} \rightarrow 3+4 \sqrt{3}$ as $\delta \rightarrow 0$.

Since the truss is not square this time, we must calculate both $a_{1}^{\delta}$ and $a_{2}^{\delta}$. First, for $y \in(0, \delta / 2) \cup(2-\delta / 2,2)$,

$$
\begin{aligned}
a_{1}^{\delta}(y) & =\int_{-\sqrt{3} / 3}^{2 \sqrt{3} / 3} \int_{\sqrt{3} x_{2} / 3-2 / 3}^{2 / 3-\sqrt{3} x_{2} / 3} x_{1}^{2} d x_{1} d x_{2}-\int_{-\sqrt{3} / 3+\delta}^{2 \sqrt{3} / 3-2 \delta} \int_{\sqrt{3} x_{2} / 3-2 / 3+2 \sqrt{3} \delta / 3}^{2 / 3-2 \sqrt{3} \delta / 3-\sqrt{3} x_{2} / 3} x_{1}^{2} d x_{1} d x_{2} \\
& =\sqrt{3}\left[1-(1-\sqrt{3} \delta)^{4}\right] / 6=2 \delta-3 \sqrt{3} \delta^{2}+6 \delta^{3}-3 \sqrt{3} \delta^{4} / 2 .
\end{aligned}
$$

By a similar calculation, we also get $a_{2}^{\delta}(y)=\sqrt{3}\left[1-(1-\sqrt{3} \delta)^{4}\right] / 6$.

For $y \in(\delta / 2,2-\delta / 2)$ we must integrate $x_{\alpha}^{2}$ over the three equilateral triangles that make up $\Sigma_{\delta}(y)$. For example,

$$
\int_{2 \sqrt{3} / 3-2 \delta}^{2 \sqrt{3} / 3} \int_{\sqrt{3} x_{2} / 3-2 / 3}^{2 / 3-\sqrt{3} x_{2} / 3} x_{1}^{2} d x_{1} d x_{2}=\frac{2}{3} \int_{2 \sqrt{3} / 3-2 \delta}^{2 \sqrt{3} / 3}\left(\frac{2}{3}-\sqrt{3} x_{2} / 3\right)^{3} d x_{2}=8 \sqrt{3} \delta^{4} / 27 .
$$

The integral over each of the other triangles is $\frac{4 \sqrt{3}}{27}\left(9 \delta^{2}-12 \sqrt{3} \delta^{3}+14 \delta^{4}\right)$. Thus, $a_{1}^{\delta}(y)=8 \sqrt{3}\left(3 \delta^{2}-4 \sqrt{3} \delta^{3}+5 \delta^{4}\right) / 9$. Again, we get the same result for $a_{2}^{\delta}$. Hence (dropping the $\alpha$ since $a_{1}^{\delta}=a_{2}^{\delta}$ ), we get

$$
\delta^{2}\left(q_{\delta}\right)^{-1}=\frac{1}{2}\left[\frac{\delta^{2}}{2-3 \sqrt{3} \delta+6 \delta^{2}-3 \sqrt{3} \delta^{3} / 2}+\frac{9(2-\delta)}{24 \sqrt{3}-96 \delta+40 \sqrt{3} \delta^{2}}\right],
$$


which tends to $\sqrt{3} / 8$ as $\delta \rightarrow 0$. Thus, for the triangular truss, the limit equation is

$$
\frac{16 \sqrt{3}}{3+4 \sqrt{3}} E I V^{\prime \prime \prime \prime}=\rho A \omega^{2} V
$$

where now $I=\int_{\omega^{e}}\left(x_{1}^{e}\right)^{2} d x_{1}^{e} d x_{2}^{e}=\int_{\omega^{e}}\left(x_{2}^{e}\right)^{2} d x_{1}^{e} d x_{2}^{e}=\sqrt{3} e^{4} / 6$ and $A=\left|\omega^{e}\right|=\sqrt{3} e^{2}$. The time $q^{*} \approx 2.7913$.

4. Concluding remarks. As noted by other authors (see [3] for example), the techniques used in Sec. 2 provide a justification for the standard theory of lowerdimensional elastic structures (in this case Euler-Bernoulli beams) without making the usual a priori assumptions. We have derived an equation of the same form as the Euler-Bernoulli beam equation but with one additional parameter (the $q^{*}$ of Sec. 3.2.1) whose value depends on the geometry of the cross section and is easily calculated.

As we remarked at the end of Sec. 2, the theory agrees, at least qualitatively, with data obtained in experiments with trusses at NASA Langley Research Center. (This will be discussed in detail in a forthcoming paper.) The predicted frequencies, however, are higher than those observed for an actual truss. This is not an unexpected result, though, because we have considered the undamped system, and the effect of damping is to lower the frequencies. We next plan to investigate several damping models (beginning with Kelvin-Voigt) using the same techniques exploited in this paper.

\section{REFERENCES}

[1] A. Bensoussan, J. L. Lions, and G. Papanicolau, Asymptotic Analysis for Periodic Structures, NorthHolland, Amsterdam, 1978

[2] P. G. Ciarlet, Mathematical Elasticity, Vol. 1: Three-Dimensional Elasticity, North-Holland, Amsterdam, 1988

[3] P. G. Ciarlet, Plates and Junctions in Elastic Multi-Structures: An Asymptotic Analysis, Collection Recherches en Mathématiques Appliquées, vol. 14, Masson, Paris, 1990

[4] P. G. Ciarlet and S. Kesavan, Two-dimensional approximations of three-dimensional eigenvalue problems in plate theory, Comput. Methods Appl. Mech. Engrg. 26, 145-172 (1981)

[5] A. Cimetière et al., Asymptotic theory and analysis for displacements and stress distribution in nonlinear elastic straight slender rods, J. Elasticity 19, 111-161 (1988)

[6] D. Cioranescu and J. Saint Jean Paulin, Homogenization in open sets with holes, J. Math. Anal. Appl. 71, 590-607 (1979)

[7] D. Cioranescu and J. Saint Jean Paulin, Reinforced and honey-comb structures, J. Math. Pures Appl. 65, 403-422 (1986)

[8] D. Cioranescu and J. Saint Jean Paulin, Elasticity problems for towers, preprint

[9] D. Cioranescu and J. Saint Jean Paulin, Towers and cranes in linearized elasticity: An asymptotic study, preprint

[10] S. Kesavan, Homogenization of elliptic eigenvalue problems. 1, Appl. Math. Optim. 5, 153-167 (1979)

[11] J. L. Lions, Some Methods in the Mathematical Analysis of Systems and Their Control, Gordon and Breach, Science Press, Bejing and New York, 1981

[12] J. Sanchez-Hubert and E. Sanchez-Palencia, Vibration and Coupling of Continuous Systems: Asymptotic Methods, Springer-Verlag, Berlin, 1989 
[13] E. Sanchez-Palencia, Non-Homogeneous Media and Vibration Theory, Lecture Notes in Phys., vol. 127, Springer-Verlag, Berlin, 1980

[14] E. Sanchez-Palencia and A. Zaoui, eds., Homogenization Techniques for Composite Media, Lecture Notes in Phys., vol. 272, Springer-Verlag, Berlin, 1987

[15] H. F. Weinberger, Variational Methods for Eigenvalue Approximation, SIAM, Philadelphia, PA, 1974

[16] J. Wloka, Partial Differential Equations, Cambridge Univ. Press, Cambridge, 1987 